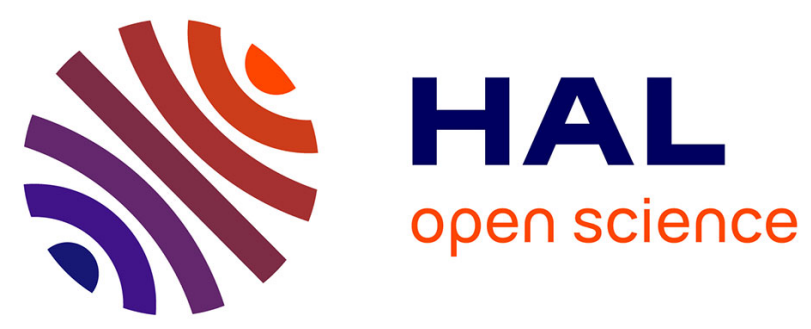

\title{
BRILLOUIN SCATTERING AT INTERFACES AND LONGWAVELENGTH ACOUSTIC PHONONS
}

\author{
V. Bortolani, F. Nizzoli, G. Santoro
}

\section{To cite this version:}

V. Bortolani, F. Nizzoli, G. Santoro. BRILLOUIN SCATTERING AT INTERFACES AND LONGWAVELENGTH ACOUSTIC PHONONS. Journal de Physique Colloques, 1984, 45 (C5), pp.C5-45C5-53. 10.1051/jphyscol:1984505 . jpa-00224113

\section{HAL Id: jpa-00224113 https://hal.science/jpa-00224113}

Submitted on 1 Jan 1984

HAL is a multi-disciplinary open access archive for the deposit and dissemination of scientific research documents, whether they are published or not. The documents may come from teaching and research institutions in France or abroad, or from public or private research centers.
L'archive ouverte pluridisciplinaire HAL, est destinée au dépôt et à la diffusion de documents scientifiques de niveau recherche, publiés ou non, émanant des établissements d'enseignement et de recherche français ou étrangers, des laboratoires publics ou privés. 


\title{
BRILLOUIN SCATTERING AT INTERFACES AND LONGWAVELENGTH ACOUSTIC PHONONS
}

\author{
V. Bortolani, F. Nizzoli and G. Santoro \\ Dipartimento di Fisica and GNSM-CNR, Università di Modena, 41100 Modena, \\ Italy
}

\begin{abstract}
Résumé - Nous présentons la thêorie de la diffusion Brillouin pour une interface entre un milieu semi-infini et un film. Suivant l'epaisseurdu film et la nature des deux milieux, la section efficace de diffusion présente différents aspects intéressants. Dans les expëriences de diffusion vers 1 'arrière que nous considérons, on trouve des structures correspondant aux ondes de Rayleigh, Sezawa et Lamb, polarisẽes dans le plan sagittal. En considérant des films transparents suffisamment épais, on peut obtenir des informations sur le mode d'interface, ou onde de Stoneley. Ce mode donne lieu à un maximum dans la section efficace Brillouin. Lorsque 1 'épaisseur du film tend vers 1 'infini, les modes de Lamb donnent la densité d'états du milieu supporté semi-infini.

Abstract - We present the theory of Brillouin scattering for an interface com posed by a semiinfinite medium and a finite slab. According to the thickness of the slab and the nature of the two systems various interesting features are present in the scattering cross section. In backward scattering experiments, to which we refer, are present structures corresponding to the Rayleigh, Sezawa and Lamb modes polarized in the sagittal plane. By considering transparent thick films of sufficiently high thickness it is possible to obtain information on the interface mode, the Stonely wave. This mode gives rise to a maximum in the Brillouin cross section. In the limit in which the thickness of the slab goes to infinity, the Lamb modes give rise to the density of states of the supported seminfinite medium.
\end{abstract}

\section{i - INTRODUCTION}

The study of surface excitations in the longwavelength limit is becoming of considera ble interest in connection with the developements of the experimental techniques. The ultrasonic measurements $/ 1 /$ allow to investigate single surface excitations by using an interdigital transducer technique. The Briliouin scattering /2-4/ gives information on the phonon field in thermal equilibrium present at the surface of a solid. In the range of frequencies investigated by this high-resolution technique (GHz region) the scattering cross section exibits interesting features associated with surfa ce and bulk phonons. For a coated system, formed by a semi infinite medium covered with a film of a different material, it is possible /5/ to observe peaks associated with Rayleigh, Sezawa, Lamb and Love modes and, for a sufficiently high thick film, the interface mode, the so called Stonely wave $/ 6 /$.

In this paper we study the properties of the Brillouin cross section of a coated system as a function of the thickness $d$ of the $f i l m$, in order to investigate the relative importance of these excitations. We review in Sect. 2 the theory of surface

Brillouin scattering for a supported film. The theory is appiied to systems of interest and the calculations are presented and discussed in sect. 3 for various thicknes ses of the film.

In the case of transparent fjlms of sufficiently high thickness we show that it is possible to detect with backward in-plane TM-TM Brillouin scattering experiments the Stonely wave. For opaque coatings the Brillouin cross section is dominated by the Rayleigh, Sezawa and Lamb waves and for high thickness of the film the Sezawa wave becomes the Stonely mode. In the limit of the film thickness going to infinity, the contribution of the Lamb modes to the cross section becomes that of the continuum of mixed modes of the seminfinite medium of film material. 


\section{2 - BRILLOUIN SCATTERING CROSS SECTION}

It has been previously shown /5,7-9/ that the surface Brillouin cross section is determined by two different scattering mechanisms. The first one is the elasto-optic coupling due to the modulation of the dielectric tensor caused by the thermally excited phonons present in the medium. The second mechanism, the ripple coupling, is due to the dynamical corrugation of the surface that acts as a grating with respect to the incoming light. In order to account for both interactions, the dielectric tensor in the whole space can be written in the form:

$$
\begin{aligned}
& \varepsilon_{\alpha \beta}(\vec{r}, t)=\delta_{\alpha \beta} \theta\left|z-d-c^{f}(\vec{R}, d, t)\right|+ \\
& \left.\left(\varepsilon_{0}^{f} \delta_{\alpha \beta}+\delta \varepsilon_{\alpha \beta}^{f}\right)\left\{\theta\left|z-c^{s}(\vec{R}, 0, t)\right|-\theta \mid z-d-c^{f}(\vec{R}, d, t)\right\}\right\}+ \\
& \left(\varepsilon_{0}^{s} \delta_{\alpha \beta}+\delta \varepsilon_{\alpha \beta}^{s}\right) \theta\left|c^{s}(\vec{R}, 0, t)-z\right|
\end{aligned}
$$

where $f$ refersto the $f i 7 m, s$ to the substrate and $d$ is the thickness of the film. $c^{m}(\vec{R}, z, t)$ is the dynamical corrugation of the surface relative to the medium $m . \varepsilon_{0}^{m}$ is the relative dielectric constant and $\theta(z)$ is the usual step function.

By expanding to first order in the phonon displacement field the corrugation function $c^{m}$ becomes simply the normal component of the phonon field $u_{z}^{m}(\vec{R}, z, t)$ and $\delta \varepsilon_{\alpha \beta}^{m}$ can be written as:

$$
\delta \varepsilon_{\alpha \beta}^{m}=\frac{1}{2} k_{\alpha \beta \gamma \delta}^{m}\left\{\frac{\partial u_{\gamma}^{m}}{\partial x_{\delta}}+\frac{\partial u_{\delta}^{m}}{\partial x_{\gamma}}\right\}
$$

$k^{m}$ is the elasto-optic tensor of the medium $m$.

To determine the cross section we solve perturbatively to first order in the displacements the Maxwell equations together with $\mathrm{Eq} .(1)$ in the two media. We impose the appropriate boundary conditions at the two rippled surfaces $z=0$ and $z=d$ for the fields sketched in Fig. 1.

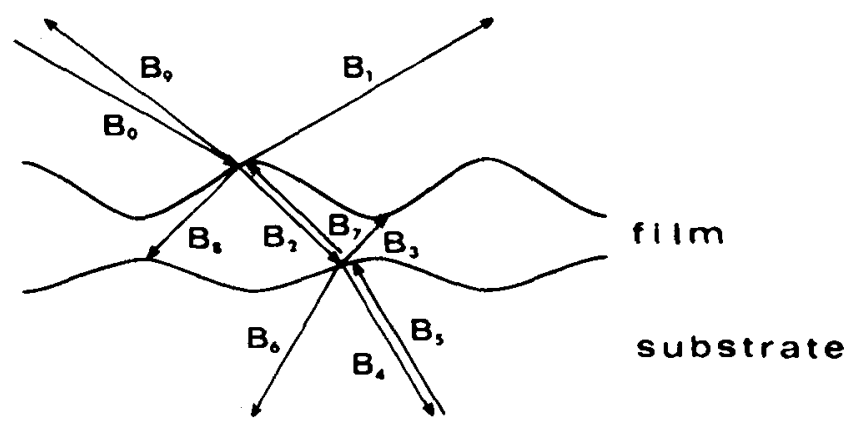

Fig. 1 - Propagation vectors and associated EM fields for a rippled coated surface. The fields $B_{0}, B_{1}, B_{2}, B_{3}$ and $B_{4}$ are zeroth-order in the displacements and satisfy the Fresnel equations. $B_{5}, B_{6}, B_{7}, B_{8}$ and $B_{9}$ are of first-order. $B_{5}$ and $B_{7}$ are relative to the polarization currents due to the modulation of the dielectric tensor in the two media. $\mathrm{B}_{9}$ is the scattered field detected in the experiments. 
The cross section is given by the thermal average on the phonon field of the normal component of the Poynting vector of the scattered field $\mathrm{B}_{9}$ divided by the normal com ponent of the Poynting vector of the incident field $B_{0}$. The details of the calculation are given in ref. $/ 5 /$. For the case of TM-TM in-plane backward scattering geome try the cross section is:

$$
\begin{aligned}
\left(\frac{\mathrm{d}^{2} \sigma}{\mathrm{d} \Omega_{\mathrm{a}} \mathrm{d} \omega}\right)_{T M-T M}= & \frac{1}{(2 \pi)^{2}}(\omega / c)^{2} \cos \theta \frac{\hbar N\left(\left|\omega-\omega_{0}\right|\right)}{2 \rho^{\mathrm{S}}\left|\omega-\omega_{0}\right|} \times \\
& \sum_{j}\left|D^{\text {TM-TM }}\left(\vec{K}-\vec{K}_{0}, \omega-\omega_{0}, j\right)\right|^{2}
\end{aligned}
$$

where:

$$
\begin{aligned}
D_{D}^{T M-T M}= & \frac{2 i p_{0}}{D D_{0}}\left\{\left(1-\varepsilon_{0}^{f}\right)\left(q^{f} q_{0}^{f} E^{-} E_{0}^{-}-\varepsilon_{0}^{f} K K_{0} E^{+} E_{0}^{+}\right) u_{z}^{f}\left(\vec{Q}, z=d, \Omega^{j}\right)\right. \\
& -4\left(\varepsilon_{0}^{s}-\varepsilon_{0}^{f}\right) \varepsilon_{0}^{f} q^{f} q_{0}^{f}\left(q^{s} q_{0}^{s} \varepsilon_{0}^{f}-K K_{0} \varepsilon_{0}^{s}\right) u_{z}^{s}\left(\vec{Q}, z=0, \Omega^{j}\right) \\
& -4 \varepsilon_{0}^{f} q^{f} q_{0}^{f} \int_{-\infty}^{0} e^{-i}\left(q^{s}+q_{0}^{s}\right) z\left[q^{s}\left(K_{0} \delta \varepsilon_{13}^{s}+q_{0}^{s} \delta \varepsilon_{11}^{s}\right)\right. \\
& \left.-K\left(K_{0} \delta \varepsilon_{33}^{s}+q_{0}^{s} \delta \varepsilon_{31}^{s}\right)\right] d z-\left[\alpha_{0}^{+} \alpha_{0}^{+} C\left(q^{f}, q_{0}^{f}\right)+\alpha^{+} \alpha_{0}^{-} C\left(q^{f},-q_{0}^{f}\right)\right. \\
& \left.\left.+\alpha^{-} \alpha_{0}^{+} c\left(-q^{f}, q_{0}^{f}\right)+\alpha^{-} \alpha_{0}^{-} C\left(-q^{f},-q_{0}^{f}\right)\right]\right\}
\end{aligned}
$$

$N(\Omega)$ is the Bose occupation number, $p^{S}$ is the mass density of the substrate. $\omega_{0}$ and $K_{0}$ are the frequency and the lateral component of the momentum of the incoming light, $\omega$ and $k$ refer to the same quantities for the scattered light. We have used the following definitions:

$$
\begin{aligned}
& p_{0}=\left\{\left(\omega_{0} / c\right)^{2}-K_{0}{ }^{2}\right\}^{\frac{1}{2}} \\
& q_{0}^{m}=\left\{\varepsilon_{0}^{m}\left(\omega_{0} / c\right)^{2}-k_{0}{ }^{2}\right\}^{\frac{1}{2}} \\
& \alpha_{0}^{ \pm}=\varepsilon_{0}^{S} q_{0}^{f} \pm \varepsilon_{0}^{f} q_{0}^{S} \\
& B_{0}^{ \pm}=\varepsilon_{0}^{f} p_{0} \pm q_{0}^{f} \\
& E_{0}^{ \pm}=\alpha_{0}^{+} e^{-i q_{0}^{f} d_{ \pm}}-\alpha_{0}^{-} e^{+i q_{0}^{f}} d \\
& D_{0}=\alpha_{0}^{+} \beta_{0}^{+} e^{-i q_{0}^{f} d}+\alpha_{0}^{-} \beta_{0}^{-} e^{+i q_{0}^{f} d} \\
& \begin{array}{c}
c\left(q^{f}, q_{0}^{f}\right)=\int_{0}^{d} e^{-i\left(q^{f}+q_{0}^{f}\right) z_{\left\{q^{f}\right.}\left(k_{0} \delta \varepsilon_{13}^{f}+q_{0}^{f} \delta \varepsilon_{11}^{f}\right)} \\
\left.-K\left(k_{0} \delta \varepsilon_{33}^{f}+q_{0}^{f} \delta \varepsilon_{31}^{f}\right)\right\} d z
\end{array}
\end{aligned}
$$

$p, q^{m}, \alpha^{ \pm}, \beta^{ \pm}, E^{ \pm}, D$ are similarly defined whithout the superscript "o". In the expression of $D$ (Eq. 4) is contained the phonon displacement field of the coated medium. In order to evaluate the displacements we consider a semiinfinite 
medium in the half space $z<0$ and the film in the region $0<z<d$.

The equations of motion in the two media, in the elastic approximation, are:

$$
\left\{\rho_{\Omega^{2} \delta}^{m_{\alpha \beta}}+c_{\gamma \alpha \beta \delta \delta}^{m} \frac{\partial^{2}}{\partial x_{\alpha} \partial x_{\beta}}\right\} w_{\beta}^{m}(\vec{r}, \Omega)=0
$$

where $c^{m}$ is the elastic tensor. The eigenvectors $\vec{w}^{m}$ can be written in the Bloch form:

$$
w_{\beta}^{m}(\vec{r}, \Omega)=e^{+i \vec{Q} \cdot \vec{R}} e^{+i q_{z}^{m} z} u_{\beta}^{m}\left(\vec{Q}, \Omega, q_{z}^{m}\right)
$$

$\vec{Q}$ is the lateral component of the phonon momentum and $q_{z}^{m}$ is the normal component. The $\vec{u}^{\mathrm{m}}$ are the polarization vectors of the two media.

The secular problem reduces to:

$$
\operatorname{det}\left\|\rho^{m} \Omega^{2} \delta_{\alpha \beta}+c_{\gamma \alpha \beta \delta^{m}}^{m} q_{\alpha}^{m} q_{\beta}^{m}\right\|=0
$$

where $\vec{q}^{m}=\left(\vec{Q}, q_{z}^{m}\right)$. By solving Eq. (7) at fixed $\Omega$ one obtain either real or complex $q_{z}^{m}$, labelled with $q_{z}^{m}, \lambda$. The polarization vector of the coated system can be written as a linear combination of "Bloch type" solutions $\mathrm{Eq}$. (6) retaining only those $\mathrm{q}_{\mathrm{z}}^{\mathrm{m}, \lambda}$ which give rise to travelling or evanescent waves to $z \rightarrow-\infty$. We write:

$$
w_{B}^{m, j}\left(\vec{r}, \Omega^{j}\right)=\sum_{\lambda} a_{m, \lambda}^{j}\left(\vec{Q}, \Omega^{j}\right) e^{+i \vec{Q} \cdot \vec{R}} e^{+i q_{z}^{m, \lambda} z} u_{B}^{m}\left(\vec{Q}, q_{z}^{m, \lambda}, \Omega^{j}\right)
$$

The index $j$ labels the independent solutions of Eq. (5) and the unknown coefficients $a_{m, \lambda}^{j}$ can be determined by imposing the boundary conditions at the free surface $z=d$ :

$$
\left(c_{\alpha \beta \mu \nu}^{f} \frac{\partial w_{\mu}^{f, j}}{\partial x_{\nu}}\right)_{z=d}=0
$$

and at the interface $z=0$ :

$$
\begin{aligned}
& \left(c_{\alpha \beta \mu \nu}^{s} \frac{\partial w_{\mu}^{s, j}}{\partial x_{\nu}}\right)_{z=0}=\left(c_{\alpha \beta \mu \nu}^{f} \frac{\partial w_{\mu}^{f, j}}{\partial x_{\nu}}\right)_{z=0} \\
& \left(w_{\beta}^{s, j}\right)_{z=0}=\left(w_{\beta}^{f, j}\right)_{z=0}
\end{aligned}
$$

According to the frequency $\Omega$ considered, the polarization vectors Eq. (8) can represent different modes.

For modes polarized in the sagittal plane, defined by the lateral component of the wavevector and by the normal to the surface, we have the Rayleigh wave decaying away from the surface, the Sezawa and Lamb modes which are localized only in the substrate and the Stonely wave localized at the interface and the mixed modes containing localized and travelling waves in both media and also bulk modes.

The Love modes, polarized normal to the sagittal plane, do not enter in the TM-TM in-plane backward scattering Brillouin cross section that we are considering.

The amplitude of the Rayleigh, Stonely and a particular Lamb mode as a function of $z$ are presented in Fig. 2 for the system $\mathrm{Ni}$ on fused silica for $\mathrm{d}=20000 \mathrm{~A}$. 


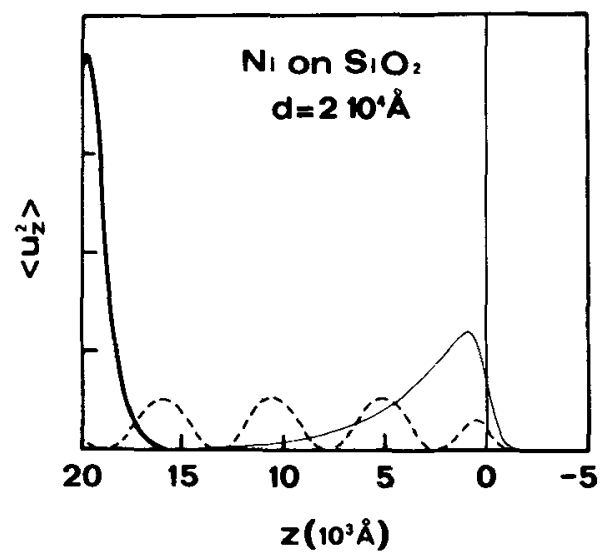

Fig. 2 - Amplitude of the thermally averaged normal component of the phonon displacement as a function of $z$.

Heavy line: Amplitude of the Rayleigh wave

Light line: Amplitude of the Stonely wave

Dashed line: Amplitude of the third Lamb wave

\section{3 - CALCULATIONS AND DISCUSSION}

In this section we apply the theory to the case of an interface between an opaque and a transparent material. For small thickness $d$ of the film, the shape of the spectrum is related to the interference between the ripple and elasto-optic couplings in the two media and can give useful information on the components of the elastooptic tensor of the supported film.

This is the case of the interface Au-Si. In Fig. 3 are reported the experimental data $111 /$ for $d=1500 \AA$. Various peaks are present due to Rayleigh, Sezawa and Lamb modes. The relative height of these peaks is related to the dielectric and elasto-optic properties of the two media. By knowning the elasto-optic properties of the substrate in the frequency range of visible light it is possible with Eqs. (3) and (4) to perform a best fit to the experimental data. In this manner one obtain the elastooptic tensor of $\mathrm{Au}$ in this frequency range where the standard techniques are not applicable. The calculated values are:

$$
k_{11}=42+i 10 \quad k_{12}=53 \quad k_{44}=-5.5+i 5
$$

With these values, the cross section (full line in Fig. 3) is in quantitative agreement with the experimental data over the whole frequency range.

The same calculations for $d=1000 \AA$ are reported in Fig. 4 and compared with experiments.

For this system, it is not possible to detect the Stonely wave because the elastic constants of the two media do not fulfill the existence condition /12/ for this wave. We pass now to consider the system $\mathrm{Ni}$-fused silica where the Stonely wave exists. First of all we study the case of a film of fused silica on a polycristalline $\mathrm{Ni}$ substrate.

In Fig. 5 is drawn the dispersion of the velocity of the surface mode as a function of the thickness $d$. For small d, the velocity of the localized mode appraches the velocity of the Rayleigh wave of clean $\mathrm{Ni}$, for $d>2000 \AA$ the localized mode becomes the Stonely wave of the system. In fact for small $\mathrm{d}$ the mode is mainly localized at 


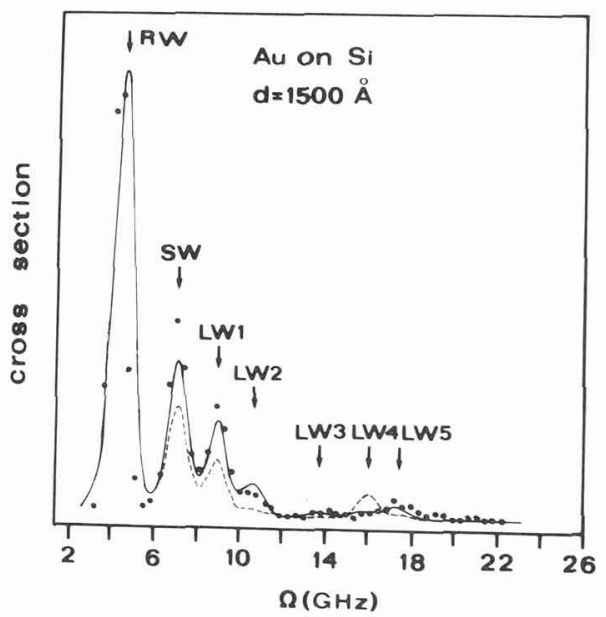

Fig. 3 - Brillouin scattering cross section of Au on Si. Dots represent the experimental data of Sandercock (private communication and paper at this conference). The experiments where performed with a laser beam of wavelength $\lambda=5745 \AA$ and the incidence angle was $=70^{\circ}$. The calculations are represented by the continuous line. The dashed 7 ine is related to the ripple calculation.

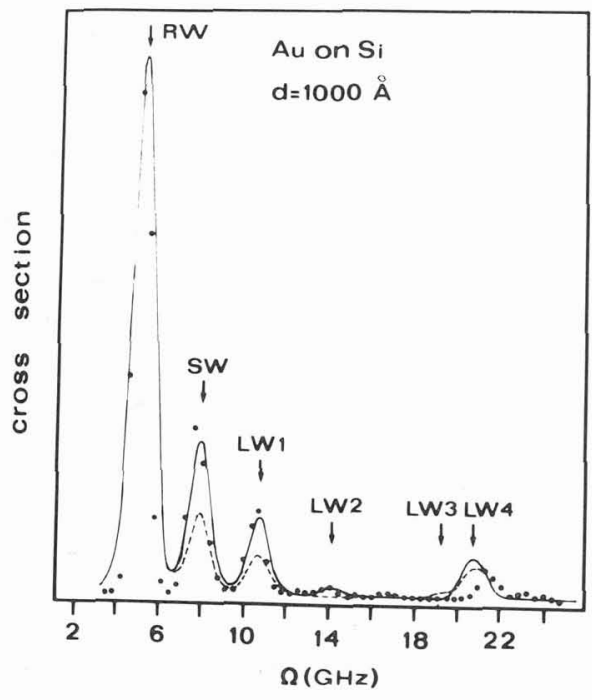

Fig. 4 - Caption as in Fig. 3 
the free surface of silica and has a small secondary maximum at the interface. By increasing $d$, the localization decreases at the surface and increases at the interface.

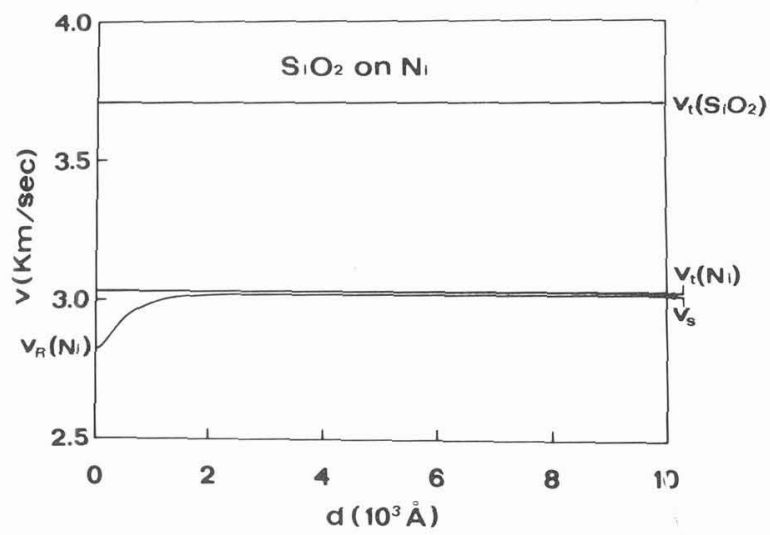

Fig. 5 - Velocity of the surface wave for $\mathrm{SiO}_{2}$ on $\mathrm{Ni}$ as a function of the $\mathrm{SiO}_{2}$ film thickness $d . v_{s}$ is the velocity of the Stonely wave. The two light horizontal lines represent the shear velocities of $\mathrm{Ni}$ and $\mathrm{SiO}_{2}$.

The evaluated cross sections for $d=500 \AA$ and $D=5000 \AA$ are drawn in Fig. 6 together with the cross section of clean silica.

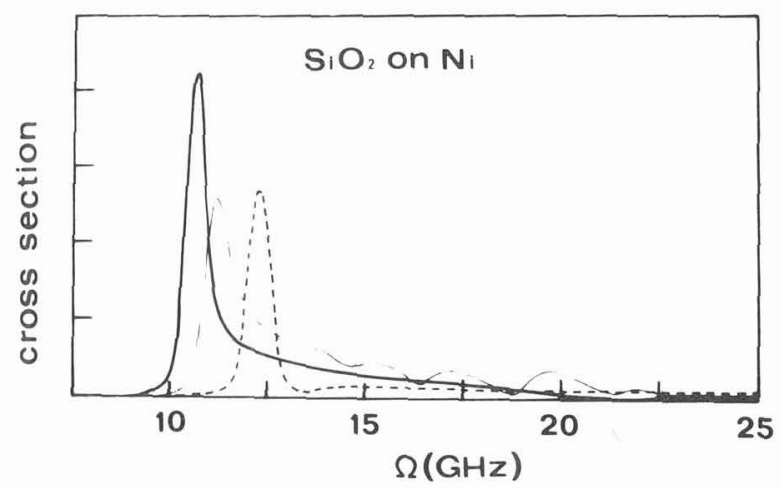

Fig. 6 - Brillouin scattering cross section for silica on nickel.

Heavy line: calculation for $\mathrm{d}=500 \AA$

Light Tine: calculation for $d=5000 \AA$

Dashed line: calculation for clean silica

In both cases the cross section is dominated by the elasto-optic coupling in the film and the ripple at the interface. We have neglected in the calculation the elasto-optic coupling in the $\mathrm{Ni}$ substrate because the very small penetration depth 
of the light in this medium.

One observes a peak associated with a localized mode and a continuum of mixed and bulk modes. For $d=500 \AA$ the peak is due to the Rayleigh wave while for $d=5000 \AA$ it is associated with the Stonely wave. Even for very large $d$ the cross section of this system remainsdistinct from the $\mathrm{SiO}_{2}$ cross section because of the different boundary conditions of the two systems.

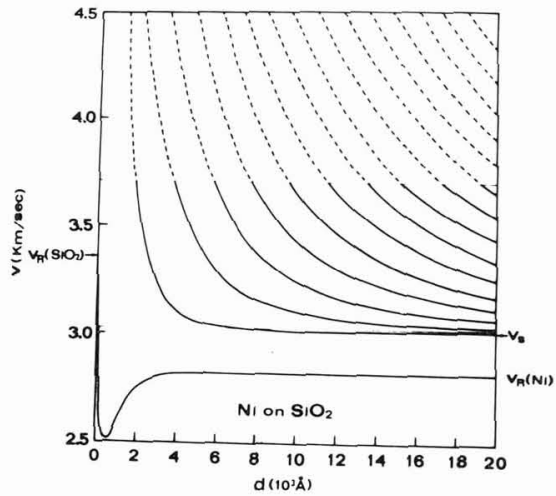

Fig. 7 - Dispersion of the velocities of the localized modes for the system Ni on silica as a function of the $\mathrm{Ni}$ film thickness. Horizontal 1 ines as in Fig. 5. The dashed curves are resonances associated with the Sezawa and Lamb modes.

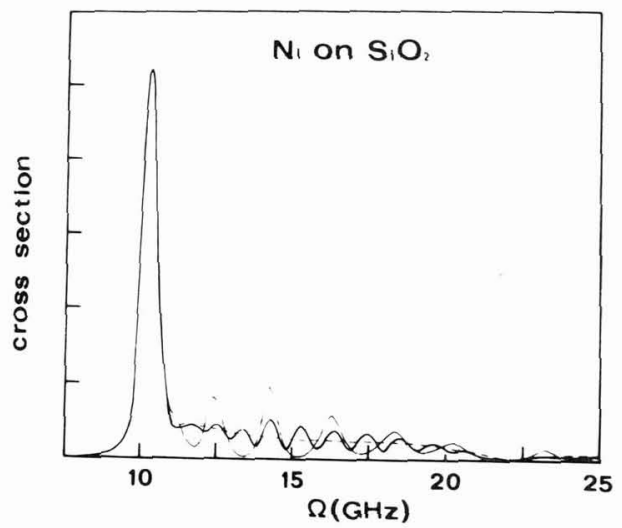

Fig. 8 - Brillouin scattering cross section for $\mathrm{Ni}$ on $\mathrm{SiO}_{2}$. Light line: calculation for $d=5000 \AA$ Heavy line: calculation for $d=10000 \AA$ Dashed line: calculation for clean Ni

The system $\mathrm{Ni}$ on fused silica is also of interest. In this case the main scattering mechanism is the ripple at the film surface. As it can be seen from Fig. 7 there are many localized modes and resonances. The lowest mode is the Rayleigh wave of the system. For smali d its velocity approaches the Rayleigh wave of clean silica and 
for large $d$ becomes that of pure nickel. The second localized mode, resonant for $d<1900 \AA$ is the Sezawa wave. This mode becomes the Stonely wave for $d>7500 \AA$; The other localized modes are the Lamb waves of this system. Their number increases as the thickness of the film increases.

The cross sections for two different values of $d$ are presented in Fig. 8 . For $d=5000 \AA$ (light line) is present the Rayleigh peak, the small shoulder on the right of it related to the Sezawa wave and the other maxima associated with the first Lamb mode and to the otber resonant Lamb modes.

In the case of $d=10000 \AA$ the number of oscillations in the cross section increases, their amplitude decreases and the cross section approaches that of clean $\mathrm{Ni}$ (dashed line in Fig. 8). We notice that for this system the Stonely wave does notproduce any significant structure in the Brillouin cross section. This is due to the small ampli tude of the ripple at the film surface caused by the Stonely wave.

In conclusion we have shown that the Brillouin scattering can be conveniently used in studying the interface modes of supported transparent films.

\section{ACKNOWLEDGMENTS}

We like to thank J.R.Sandercock for useful discussions and for making available to us his preliminary unpublished measurements for the Au-Si system.

\section{REFERENCES}

1 - AULD B.A., "Acoustic fields and waves in solids" vol.II (New York: WileyInterscience 1973)

2 - SANDERCOCK J.R., Solid State Commun. 26 (1978) 547

3 - HARTLEY R.T. and FLEURY P.A., J.Phys. C: Solid State Phys. 12 (1979) L863

4 - MISHRA S. and BRAY R., Phys.Rev.Lett. 39 (1977) 222

5 - BORTOLANI V., MARVIN M., NIZZOLI F. and SANTORO G., J.Phys. C: Solid State Phys. 16 (1983) 1757

6 - FARNELL G.W. and ADLER E.L., Physical Acoustic Principle and Methods vol. 9 ed. MASON W.P. and THURSTON R.N. (New York: Academic Press 1972) p.35

7 - SUBBASWAMY K.R. and MARADUDIN A.A., Phys.Rev. B18 (1978) 4181

8 - ROWELL N.L. and STEGEMAN G.I., Phys.Rev. B18 (1978) 2598

9 - LOUDON R., J.Phys. C: Solid State Phys. $1 T(1978) 2623$

10 - BORTOLANI V., NIZZOLI F., SANTORO G. and SANDERCOCK J.R., Phys.Rev. B25 (1982) 3442

11 - SANDERCOCK J.R., Paper at this Conference

12 - DJAFARI-ROUHANI B., DOBRZYNSKI L. and MASRI P., Ann.Phys. FR $\underline{6}$ (1981) 859 